\title{
Depth reduction of a class of Witten zeta functions
}

\author{
Xia Zhou* \\ Department of mathematics \\ Zhejiang University \\ Hangzhou, 310027 \\ P.R.China \\ xiazhou0821@hotmail.com
}

\author{
David M. Bradley \\ Department of Mathematics \& Statistics \\ University of Maine \\ 5752 Neville Hall Orono, Maine 04469-5752 \\ U.S.A \\ bradley@math.umaine.edu, dbradley@member.ams.org \\ Tianxin Cai \\ Department of mathematics \\ Zhejiang University \\ Hangzhou, 310027 \\ P.R.China \\ caitianxin@hotmail.com
}

Submitted: Apr 6, 2008; Accepted: Jul 21, 2009; Published: Jul 31, 2009

Mathematics Subject Classifications: 11A07, 11A63

\begin{abstract}
We show that if $a, b, c, d, f$ are positive integers such that $a+b+c+d+f$ is even, then the Witten zeta value $\zeta_{\mathfrak{s l}(4)}(a, b, c, d, 0, f)$ is expressible in terms of Witten zeta functions with fewer arguments.
\end{abstract}

\section{Introduction}

Let $\mathbf{N}$ be the set of positive integers, $\mathbf{Q}$ the field of rational numbers, $\mathbf{C}$ the field of complex numbers.

For any semisimple Lie algebra $\mathfrak{g}$, the Witten zeta function(cf. [5]) is defined by

$$
\zeta_{\mathfrak{g}}(s)=\sum_{\rho}(\operatorname{dim} \rho)^{-s}
$$

where $s \in \mathbf{C}$ and $\rho$ runs over all finite dimensional irreducible representations of $\mathfrak{g}$. In order the calculate the volumes of certain moduli space, Witten [7] introduced the values

${ }^{*}$ The first and third authors are supported by the National Natural Science Foundation of China, Project 10871169 . 
$\zeta_{\mathfrak{g}}(2 k)$ for $k \in \mathbf{N}$ and showed that $\pi^{-2 k l} \zeta_{\mathfrak{g}}(2 k) \in \mathbf{Q}$, where $l$ is the number of positive roots of $\mathfrak{g}$.

For positive integer $r$, Matsumoto and Tsumura [5] defined a multi-variate extension, called the Witten multiple zeta-function associated with $\mathfrak{s l}(r+1)$, by

$$
\zeta_{\mathfrak{s l}(r+1)}(\mathbf{s})=\sum_{m_{1}, \ldots, m_{r}=1}^{\infty} \prod_{j=1}^{r} \prod_{k=1}^{r-j+1}\left(\sum_{v=k}^{j+k-1} m_{v}\right)^{-s_{j, k}}
$$

where

$$
\mathbf{s}=\left(s_{j, k}\right)_{1 \leq j \leq r ; 1 \leq k \leq r-j+1} \in \mathbf{C}^{r(r+1) / 2}, \quad \Re\left(s_{j, k}\right)>1 .
$$

In particular ([5], section 2, Prop 2.1), if $m \in \mathbf{N}$ we denote

$$
\zeta_{\mathfrak{s l}(r+1)}(2 m):=\prod_{1 \leq j<k \leq r+1}(k-j) \zeta_{\mathfrak{s l}(r+1)}(\underbrace{2 m, \ldots, 2 m}_{r(r+1) / 2}) .
$$

As in [1], given the Witten multiple zeta-function (1), we define the depth to be $r$. Further, if the zeta functions $y_{1}, \ldots, y_{k}$ have depth $r_{1}, \ldots, r_{k}$ respectively, then for $a_{1}, \ldots, a_{k} \in \mathbf{C}$, we define the depth of $a_{1} y_{1}+\cdots+a_{k} y_{k}$ to be $\max \left\{r_{i}: 1 \leq i \leq k\right\}$. We would like to know which sums can be expressed in terms of lower depth sums. When a sum can be so expressed, we say it is reducible.

An explicit evaluation for $\zeta_{\mathfrak{s r}(3)}(2 m)(m \in \mathbf{N})$ was independently discovered by D. Zagier, S. Garoufalidis, and L. Weinstein (see [8, page 506]). In [3], Gunnells and Sczech provided a generalization of the continued-fraction algorithm to compute high-dimensional Dedekind sums. As examples, they gave explicit evaluations of $\zeta_{\mathfrak{s l}(3)}(2 m)$ and $\zeta_{\mathfrak{s l}(4)}(2 m)$. Matsumoto and Tsumura [5] considered functional relations for Witten multiple zetafunctions, and found that

$$
\begin{aligned}
(-1)^{a} \zeta_{\mathfrak{s l}(4)}\left(s_{1}, s_{2}, a, s_{3}, 0, b\right)+(-1)^{b} \zeta_{\mathfrak{s l}(4)}\left(s_{1}, s_{2}, b, s_{3}, 0, a\right) & \\
& +\zeta_{\mathfrak{s l}(4)}\left(a, 0, s_{2}, s_{1}, b, s_{3}\right)+\zeta_{\mathfrak{s l}(4)}\left(b, 0, s_{1}, s_{2}, a, s_{3}\right)
\end{aligned}
$$

is reducible for any $a, b \in \mathbf{N}$ and $s_{1}, s_{2}, s_{3} \in \mathbf{C}$.

In this paper, we provide a combinatorial method which gives a simpler formula for the quantity (2). Furthermore, we show that if $a, b, c, d, f$ are positive integers such that $a+b+c+d+f$ is even, then $\zeta_{\mathfrak{s l}(4)}(a, b, c, d, 0, f)$ is reducible.

\section{Functional relation}

Lemma 2.1. If the function $F: \mathbf{Z}_{\geq 0} \times \mathbf{Z}_{\geq 0} \times \mathbf{C} \rightarrow \mathbf{C}$ has the property that there exist $p, q \in \mathbf{C}$ such that for every $a, b \in \mathbf{N}$ and every $s \in \mathbf{C}$ the relation

$$
F(a, b, s)=p F(a-1, b, s+1)+q F(a, b-1, s+1)
$$


holds, then for every $a, b \in \mathbf{N}$ and every $s \in \mathbf{C}$,

$$
\begin{aligned}
F(a, b, s) & =\sum_{j=1}^{b} p^{a} q^{b-j}\left(\begin{array}{c}
a+b-j-1 \\
a-1
\end{array}\right) F(0, j, a+b+s-j) \\
& +\sum_{j=1}^{a} p^{a-j} q^{b}\left(\begin{array}{c}
a+b-j-1 \\
b-1
\end{array}\right) F(j, 0, a+b+s-j) .
\end{aligned}
$$

Proof. It's easy to prove Lemma 2.1 by induction.

The Euler sum of depth $r$ and weight $w$ is a multiple series of the form

$$
\zeta\left(s_{1}, \ldots, s_{r}\right):=\sum_{n_{1}>\cdots>n_{r}>0} \prod_{j=1}^{r} n_{j}^{-s_{j}}
$$

with weight $w:=s_{1}+\cdots+s_{r}$. Now let's recall the following result concerning the reduction on the triple Euler sums.

Lemma 2.2 (Borwein-Girgensohn [2]). Let $a, b, c$ be positive integers. If $a+b+c$ is even or less than or equal to 10 , then $\zeta(a, b, c)$ can be expressed as a rational linear combination of products of single and double Euler sums of weight $a+b+c$.

Lemma 2.3 (Huard-Williams-Zhang [4]). If $a, b, c$ be positive integers, then

$$
\zeta_{\mathfrak{s l}(3)}(a, b, c)=\left\{\sum_{j=1}^{a}\left(\begin{array}{c}
a+b-j-1 \\
b-1
\end{array}\right)+\sum_{j=1}^{b}\left(\begin{array}{c}
a+b-j-1 \\
a-1
\end{array}\right)\right\} \zeta(a+b+c-j, j) .
$$

Moreover, $\zeta_{\mathfrak{s l}(3)}(a, b, c)$ can be explicitly evaluated in terms of the values of Riemann zeta functions when $a+b+c$ is odd.

Theorem 2.1. If $a, b \in \mathbf{N}$, then

$$
\begin{aligned}
& (-1)^{a} \zeta_{\mathfrak{s l}(4)}\left(s_{1}, s_{2}, a, s_{3}, 0, b\right)+(-1)^{b} \zeta_{\mathfrak{s l}(4)}\left(s_{1}, s_{2}, b, s_{3}, 0, a\right) \\
& +\zeta_{\mathfrak{s l}(4)}\left(a, 0, s_{2}, s_{1}, b, s_{3}\right)+\zeta_{\mathfrak{s l}(4)}\left(b, 0, s_{1}, s_{2}, a, s_{3}\right) \\
& =\sum_{i=1}^{\max (a, b)}\left\{\left(\begin{array}{c}
a+b-i-1 \\
a-1
\end{array}\right)+\left(\begin{array}{c}
a+b-i-1 \\
b-1
\end{array}\right)\right\}(-1)^{i} \zeta(i) \\
& \times \zeta_{\mathfrak{s l}(3)}\left(s_{1}, s_{2}, s_{3}+a+b-i\right) \\
& +\sum_{i=1}^{a}\left(\begin{array}{c}
a+b-i-1 \\
b-1
\end{array}\right)\left\{\zeta(i) \zeta_{\mathfrak{s l}(3)}\left(s_{1}, s_{2}, s_{3}+a+b-i\right)\right. \\
& \left.-\zeta_{\mathfrak{s l}(3)}\left(s_{1}+i, s_{2}, s_{3}+a+b-i\right)-\zeta_{\mathfrak{s l}(3)}\left(s_{1}, s_{2}, s_{3}+a+b\right)\right\}
\end{aligned}
$$




$$
\begin{aligned}
+\sum_{i=1}^{b}\left(\begin{array}{c}
a+b-i-1 \\
a-1
\end{array}\right)\left\{\zeta(i) \zeta_{\mathfrak{s l}(3)}\left(s_{1}, s_{2}, s_{3}+a+b-i\right)\right. \\
\left.\quad-\zeta_{\mathfrak{s l}(3)}\left(s_{2}+i, s_{1}, s_{3}+a+b-i\right)-\zeta_{\mathfrak{s l}(3)}\left(s_{1}, s_{2}, s_{3}+a+b\right)\right\} .
\end{aligned}
$$

Proof. From the definition (1) of the Witten multiple zeta-function, we have

$$
\zeta_{\mathfrak{s l}(4)}\left(s_{1}, s_{2}, s_{3}, s_{4}, s_{5}, s_{6}\right)=\zeta_{\mathfrak{s l}(4)}\left(s_{3}, s_{2}, s_{1}, s_{5}, s_{4}, s_{6}\right) .
$$

Next, for any $a, b \in \mathbf{N}$ and $s_{1}, s_{2}, s_{3} \in \mathbf{C}$, since

$$
\zeta_{\mathfrak{s l}(4)}\left(s_{1}, s_{2}, a, s_{3}, 0, b\right)=\zeta_{\mathfrak{s l}(4)}\left(s_{1}, s_{2}, a, s_{3}+1,0, b-1\right)-\zeta_{\mathfrak{s l}(4)}\left(s_{1}, s_{2}, a-1, s_{3}+1,0, b\right),
$$

by Lemma 2.1, we have

$$
\begin{aligned}
\zeta_{\mathfrak{s l}(4)}\left(s_{1}, s_{2}, a, s_{3}, 0, b\right) & =\sum_{i=1}^{a}\left(\begin{array}{c}
a+b-i-1 \\
b-1
\end{array}\right)(-1)^{a+i} \zeta_{\mathfrak{s l}(4)}\left(s_{1}, s_{2}, i, s_{3}+a+b-i, 0,0\right) \\
& +\sum_{i=1}^{b}\left(\begin{array}{c}
a+b-i-1 \\
a-1
\end{array}\right)(-1)^{a} \zeta_{\mathfrak{s l}(4)}\left(s_{1}, s_{2}, 0, s_{3}+a+b-i, 0, i\right) .
\end{aligned}
$$

Similarly, we have

$$
\begin{aligned}
\zeta_{\mathfrak{s l}(4)}\left(s_{1}, s_{2}, b, s_{3}, 0, a\right) & =\sum_{i=1}^{b}\left(\begin{array}{c}
a+b-i-1 \\
a-1
\end{array}\right)(-1)^{b+i} \zeta_{\mathfrak{s l}(4)}\left(s_{1}, s_{2}, i, s_{3}+a+b-i, 0,0\right) \\
& +\sum_{i=1}^{a}\left(\begin{array}{c}
a+b-i-1 \\
b-1
\end{array}\right)(-1)^{b} \zeta_{\mathfrak{s l}(4)}\left(s_{1}, s_{2}, 0, s_{3}+a+b-i, 0, i\right), \\
\zeta_{\mathfrak{s l}(4)}\left(a, 0, s_{2}, s_{1}, b, s_{3}\right)= & \sum_{i=1}^{a}\left(\begin{array}{c}
a+b-i-1 \\
b-1
\end{array}\right) \zeta_{\mathfrak{s l}(4)}\left(i, 0, s_{2}, s_{1}, 0, s_{3}+a+b-i\right) \\
& +\sum_{i=1}^{b}\left(\begin{array}{c}
a+b-i-1 \\
a-1
\end{array}\right) \zeta_{\mathfrak{s l}(4)}\left(0,0, s_{2}, s_{1}, i, s_{3}+a+b-i\right),
\end{aligned}
$$

and

$$
\begin{aligned}
\zeta_{\mathfrak{s l}(4)}\left(b, 0, s_{1}, s_{2}, a, s_{3}\right) & =\sum_{i=1}^{b}\left(\begin{array}{c}
a+b-i-1 \\
a-1
\end{array}\right) \zeta_{\mathfrak{s l}(4)}\left(i, 0, s_{1}, s_{2}, 0, s_{3}+a+b-i\right) \\
& +\sum_{i=1}^{a}\left(\begin{array}{c}
a+b-i-1 \\
b-1
\end{array}\right) \zeta_{\mathfrak{s l}(4)}\left(0,0, s_{1}, s_{2}, i, s_{3}+a+b-i\right) .
\end{aligned}
$$


Since

$$
\begin{aligned}
\zeta_{\mathfrak{s l}(4)}(a, b, c, d, 0,0) & =\zeta(c) \zeta_{\mathfrak{s l}(3)}(a, b, d), \\
\zeta_{\mathfrak{s l}(4)}(a, b, 0, c, 0, d) & =\sum_{\substack{n_{1}, n_{2}=1 \\
v>n_{1}+n_{2}}} \frac{1}{v^{d} n_{1}^{a} n_{2}^{b}\left(n_{1}+n_{2}\right)^{c}} \\
& =\sum_{\substack{n_{1}, n_{2}=1 \\
v>n_{1}+n_{2}}} \frac{1}{v^{d} n_{1}^{b} n_{2}^{a}\left(n_{1}+n_{2}\right)^{c}}, \\
\zeta_{\mathfrak{s l}(4)}(a, 0, b, c, 0, d) & =\sum_{\substack{n_{1}, n_{2}=1 \\
v<n_{1}}} \frac{1}{v^{a} n_{1}^{c} n_{2}^{b}\left(n_{1}+n_{2}\right)^{d}}, \\
\zeta_{\mathfrak{s l}(4)}(0,0, a, b, c, d) & =\sum_{\substack{n_{1}, n_{2}=1 \\
n_{1}+n_{2}>v>n_{1}}} \frac{1}{v^{c} n_{1}^{a} n_{2}^{b}\left(n_{1}+n_{2}\right)^{d}},
\end{aligned}
$$

we find that

$$
\begin{aligned}
\zeta_{\mathfrak{s l}(4)}\left(s_{1}, s_{2}, 0, s_{3}+a+\right. & b-i, 0, i)+\zeta_{\mathfrak{s l}(4)}\left(i, 0, s_{2}, s_{1}, 0, s_{3}+a+b-i\right) \\
& +\zeta_{\mathfrak{s l}(4)}\left(0,0, s_{1}, s_{2}, i, s_{3}+a+b-i\right) \\
=\zeta(i) \zeta_{\mathfrak{s l}(3)}\left(s_{1}, s_{2}, s_{3}+\right. & a+b-i)-\zeta_{\mathfrak{s l}(3)}\left(s_{1}+i, s_{2}, s_{3}+a+b-i\right) \\
& -\zeta_{\mathfrak{s l}(3)}\left(s_{1}, s_{2}, s_{3}+a+b\right)
\end{aligned}
$$

and

$$
\begin{aligned}
\zeta_{\mathfrak{s l}(4)}\left(s_{1}, s_{2}, 0, s_{3}+a+\right. & b-i, 0, i)+\zeta_{\mathfrak{s l}(4)}\left(i, 0, s_{1}, s_{2}, 0, s_{3}+a+b-i\right) \\
& +\zeta_{\mathfrak{s l}(4)}\left(0,0, s_{2}, s_{1}, i, s_{3}+a+b-i\right) \\
=\zeta(i) \zeta_{\mathfrak{s l}(3)}\left(s_{1}, s_{2}, s_{3}+\right. & a+b-i)-\zeta_{\mathfrak{s l}(3)}\left(s_{2}+i, s_{1}, s_{3}+a+b-i\right) \\
& -\zeta_{\mathfrak{s l}(3)}\left(s_{1}, s_{2}, s_{3}+a+b\right)
\end{aligned}
$$

Now combining equations (8-17), we complete the proof.

Lemma 2.4. Every Witten multiple zeta value of the form $\zeta_{\mathfrak{s l}(4)}(a, b, 1, d, 0,1)$ with $a, b, d \in$ $\mathbf{N}$ can be expressed as a rational linear combination of products of single and double Euler sums when $a+b+d$ is even or $a+b+d \leq 8$.

Proof.

$$
\begin{aligned}
\zeta_{\mathfrak{s l}(4)}(a, b, 1, d, 0,1) & =\sum_{i=1}^{a}\left(\begin{array}{c}
a+b-i-1 \\
b-1
\end{array}\right) \zeta_{\mathfrak{s l}(4)}(i, 0,1, a+b+d-i, 0,1) \\
& +\sum_{i=1}^{b}\left(\begin{array}{c}
a+b-i-1 \\
a-1
\end{array}\right) \zeta_{\mathfrak{s l}(4)}(0, i, 1, a+b+d-i, 0,1) .
\end{aligned}
$$


However, for any $a, d \in \mathbf{N}$,

$$
\begin{aligned}
\zeta_{\mathfrak{s l}(4)}(a, 0,1, d, 0,1) & =\zeta_{\mathfrak{s l}(4)}(0, a, 1, d, 0,1) \\
& =\zeta_{\mathfrak{s l}(4)}(a, 0,1,0,0, d+1)+\sum_{i=1}^{d} \zeta(d+2-i, i, a),
\end{aligned}
$$

and

$$
\zeta_{\mathfrak{s l}(4)}(a, 0,1,0,0, d+1)=\zeta(d+1, a, 1)+\sum_{i=1}^{a} \zeta(d+1, a+1-i, i) .
$$

We complete the proof by combining this with Lemma 2.2 .

Theorem 2.2. Every Witten multiple zeta value of the form $\zeta_{\mathfrak{s l}(4)}(a, b, c, d, 0, f)$ with $a, b, c, d, f, \in \mathbf{N}$ can be expressed as a rational linear combination of products of single and double Euler sums when $a+b+c+d+f$ is even or $a+b+c+d+f \leq 10$.

Proof. From Lemma 2.1, we see that

$$
\begin{aligned}
& \frac{1}{n_{1}^{a} n_{2}^{b} n_{3}^{c}\left(n_{1}+n_{2}\right)^{d}\left(n_{1}+n_{2}+n_{3}\right)^{f}} \\
& =\sum_{i=1}^{c}\left(\begin{array}{c}
c+f-i-1 \\
f-1
\end{array}\right)(-1)^{c+i} \frac{1}{n_{1}^{a} n_{2}^{b} n_{3}^{i}\left(n_{1}+n_{2}\right)^{c+d+f-i}} \\
& +\sum_{i=1}^{f}\left(\begin{array}{c}
c+f-i-1 \\
c-1
\end{array}\right)(-1)^{c} \frac{1}{n_{1}^{a} n_{2}^{b}\left(n_{1}+n_{2}\right)^{c+d+f-i}\left(n_{1}+n_{2}+n_{3}\right)^{i}} \text {. }
\end{aligned}
$$

Also

$$
\begin{aligned}
& \frac{1}{n_{1}^{a} n_{2}^{b}\left(n_{1}+n_{2}\right)^{c+d+f-i}\left(n_{1}+n_{2}+n_{3}\right)^{i}} \\
& =\sum_{j=1}^{a}\left(\begin{array}{c}
a+b-j-1 \\
b-1
\end{array}\right) \frac{1}{n_{1}^{j}\left(n_{1}+n_{2}\right)^{a+b+c+d+f-i-j}\left(n_{1}+n_{2}+n_{3}\right)^{i}} \\
& \quad+\sum_{j=1}^{b}\left(\begin{array}{c}
a+b-j-1 \\
a-1
\end{array}\right) \frac{1}{n_{2}^{j}\left(n_{1}+n_{2}\right)^{a+b+c+d+f-i-j}\left(n_{1}+n_{2}+n_{3}\right)^{i}} .
\end{aligned}
$$

Now combine (20), (21) and Lemma 2.4 and sum over all ordered triples of positive integers $\left(n_{1}, n_{2}, n_{3}\right)$ to obtain

$$
\zeta_{\mathfrak{s l}(4)}(a, b, c, d, 0, f)=\sum_{i=2}^{c}\left(\begin{array}{c}
c+f-i-1 \\
f-1
\end{array}\right)(-1)^{c+i} \zeta(i) \zeta_{\mathfrak{s l}(3)}(a, b, c+d+f-i)
$$




$$
\begin{aligned}
& +\sum_{i=2}^{f}\left(\begin{array}{c}
c+f-i-1 \\
c-1
\end{array}\right)(-1)^{c}\left\{\sum_{j=1}^{a}\left(\begin{array}{c}
a+b+j-1 \\
b-1
\end{array}\right)\right. \\
& \quad \times \zeta(i, c+d+f+a+b-i-j, j) \\
& \left.+\sum_{j=1}^{b}\left(\begin{array}{c}
a+b+j-1 \\
a-1
\end{array}\right) \zeta(i, c+d+f+a+b-i-j, j)\right\} \\
& -(-1)^{c}\left(\begin{array}{c}
c+f-2 \\
c-1
\end{array}\right) \zeta_{\mathfrak{s l}(4)}(a, b, 1, c+d+f-2,0,1) .
\end{aligned}
$$

By Lemmas 2.2, 2.3 and 2.4, we complete the proof.

Remark. When $d=0$, the Witten zeta value $\zeta_{\mathfrak{s l}(4)}(a, b, c, 0,0, f)$ can also be viewed as a Mordell-Tornheim sum with depth 3 . The fact that every such sum can be expressed as a rational linear combination of products of single and double Euler sums when the weight $a+b+c+f$ is even has been shown in [6] and [9].

Acknowledgment. The authors are grateful to the referee for carefully reading the manuscript and providing several constructive suggestions.

\section{References}

[1] J. M. Browein, D. M. Bradley, D. J. Broadhurst and P. Lisonek, Special values of multiple polylogarithms, Trans. Amer. Math. Soc. 353 (2001), no. 3 p. 907-941.

[2] J. M. Borwein and R. Girgensohn, Evaluations of triple Euler sums, Electron. J. Combin., 3 (1996), no. 1, Research Paper 23, approx. 27 pp.

[3] P. E. Gunnells and R. Sczech, Evaluations of Dedekind sums, Eisenstein cocycles, and special values of $L$-functions, Duke. J. Math., 118 (2003), p. 229-260.

[4] J. G. Huard, K. S. Williams and N. Y. Zhang, On Tornheim's double series, Acta Arith., 75 (1996), no 2, 105-117. [MR 1379394] (97f:11073)

[5] K. Matsumoto and H. Tsumura, On Witten multiple zeta-functions associated with semisimple Lie Algebras I, Ann. Inst. Fourier, 56(5) (2006), p. 1457-1504.

[6] H. Tsumura, On Mordell-Tornheim zeta values, Proc. Amer. Math. Soc., 133 (2005), no. 8, 2387-2393. [MR 2138881] (2006k:11179)

[7] E. Witten, On quantum gauge theories in two dimensions, Comm. Math. Phy. 141 (1991): 153-209.

[8] D. Zagier, Values of zeta functions and their applications, in Proc. First Congress of Math.,Paris, vol. II Progress in Math., vol. 120, Birkhäuser, 1994, p. 497-512.

[9] X. Zhou, D. M. Bradley and T. Cai, On Mordell-Tornheim sums and multiple zeta values, submitted. 